\title{
PROPOSTA E IMPLEMENTAÇÃO DE SISTEMA DE SOFTWARE PARA FILMAGEM E REGISTRO DO ATENDIMENTO ODONTOLÓGICO
}

\section{PROPOSAL AND IMPLEMENTATION OF SOFTWARE SYSTEMS FOR FILMING AND REGISTRATION OF DENTAL CARE}

\author{
Antonio Carlos dos Santos Costa', Uallace da Silva Oliveira', Sérgio Donha Yarid', Alex \\ Ferreira Santos ${ }^{2}$, Suélem Maria Santana Pinheiro Ferreira ${ }^{3}$, Saulo Correa Peixoto ${ }^{1}$
}

Universidade Estadual do Sudoeste da Bahia - UESB ${ }^{1}$, Universidade Federal do Recôncavo da Bahia - UFRB ${ }^{2}$, Universidade do Estado da Bahia - UNEB ${ }^{3}$

\begin{abstract}
This paper presents a software system of dental clinic management that, in addition to complying with all requirements regarding safety, functionality and interface criteria, uses the filming feature as integral part of the dental records. All documentation related to the patient, the consultations and procedures performed, should be stored indefinitely, to protect the Dentist and patient, avoiding judicial conflict. Faced with this fact, filming is a legal document to fill potential information gaps in conventional dental documentation. For the development of the software system, the system requirements were initially raised and modeled, and after this process the first version containing the Patient Management, Employees / Users, Schedule, Treatment Plan, Odontogram and Record Query. The software system met the management requirements of a dental clinic, met the requirements of functionality, security and interface, and presented improvements compared to other software systems on the market, in addition to integrating legal filming to dental records.
\end{abstract}

Key words: Software Systems, Information Systems, Forensic Dentistry.

\section{Resumo}

Neste artigo é apresentado um sistema de software de gestão de clínica odontológica que além de cumprir com todos os requisitos quanto aos critérios de segurança, funcionalidade $e$ interface, utiliza o recurso da filmagem como parte integrante do prontuário odontológico. Todas as documentações relacionadas ao paciente, os atendimentos e procedimentos realizados, devem ser guardadas por tempo indeterminado, para proteger o CirurgiãoDentista e paciente, evitando embate judicial. Diante deste fato, a filmagem constitui documento legal para preencher possiveis lacunas de informação na documentação odontológica convencional. Para o desenvolvimento do sistema de software, inicialmente foram levantados e modelados os requisitos do sistema e após este processo foi desenvolvido a primeira versão contendo os módulos de Gerenciamento de Pacientes, Funcionários/Usuários, Agenda, Plano de Tratamento, Odontograma e Registro de Filmagem da Consulta. O sistema de software produzido atendeu aos requisitos de gestão de uma clínica odontológica, cumpriu os requisitos de funcionalidade, segurança $e$ interface, $e$ apresentou melhorias em relação aos demais sistemas de software existentes no mercado, além de integrar a filmagem legal ao prontuário odontológico.

Palavras-chave: Sistemas de Software, Sistemas de Informação, Odontologia legal. 


\section{Introdução}

Os avanços referentes à Tecnologia da Informação (TI) nos últimos anos têm permitido as empresas realizarem operações não vislumbradas anteriormente. Diante deste cenário, empresas que utilizam recursos tecnológicos se destacam em relação às outras que não fazem uso da $\mathrm{TI}^{1}$.

Para Albuquerque et al. $^{2}$, a $\mathrm{TI}$ apresenta grande importância para os profissionais da área de saúde, ajudando-os a organizar e controlar informações referentes aos seus pacientes. Além disso, a crescente sofisticação apresentada pelos sistemas de software voltados à saúde vem desempenhando um papel de destaque em minimizar ocorrências de erros nos diagnósticos, auxiliar em processos de tomada de decisão e fornecer um feedback sobre o desempenho dos procedimentos realizados ${ }^{3}$.

O surgimento e uso de aplicações de TI na área da saúde afetou de modo bastante significativo o cotidiano dos profissionais da área de Odontologia ${ }^{4}$. Para o Cirurgião-Dentista, a utilização de ferramentas informatizadas proporciona agilidade, comodidade e segurança, além de ser um instrumento de marketing que o diferencia no mercado, dando-o vantagem em um mundo cada vez mais competitivo ${ }^{5}$. Na gerência de clínica odontológica, os recursos administrativos dos sistemas de software auxiliam o profissional na criação, armazenamento, análise e retorno de informações que abrangem desde a parte financeira da clínica até documentos do prontuário odontológico de pacientes.

Apesar de existirem disponíveis no mercado diversos sistemas de software de apoio à gestão de clínicas odontológicas, um estudo sinalizou que a maioria destes apresenta falhas quanto aos critérios básicos de segurança, funcionalidade e interface ${ }^{6}$. Isso representa um fator estimulante para o desenvolvimento de um sistema de gerenciamento de clínica odontológica que contenha recursos de segurança, interface amigável e elementos indispensáveis para a composição do prontuário odontológico, conforme recomendação do Conselho Federal de Odontologia (CFO) $)^{7}$. Ademais, o recurso da filmagem como uma prova legal tem sido uma realidade no âmbito da magistratura ${ }^{8}$, e traz a possibilidade de proteger profissional e paciente em caso de embate judicial, o que o torna um produto importante a ser adicionado na gestão da clínica odontológica.

A definição de segurança, conforme a literatura9,10,11, pode ser sumarizada como a proteção contra o uso ou acesso não autorizado à informação. Diante disso, é necessário que os sistemas computacionais tenham recurso que limitem o acesso e a utilização dos documentos odontológicos somente a pessoas autorizadas.

No quesito funcionalidade, Oliveira ${ }^{6}$ em seu estudo considerou a existência de itens fundamentais (identificação do profissional, identificação do paciente, anamnese, exame clínico, plano de tratamento, evolução e intercorrências do tratamento) que compõem a ficha clínica e itens suplementares (receitas, atestados, contrato de alocação de serviços odontológicos e exames complementares), que segundo o Conselho Federal de Odontologia fazem parte da documentação odontológica ${ }^{3}$, e, portanto devem estar presentes em um sistema que presta auxílio ao profissional da Odontologia.

Quanto à interface, segundo Barbosa e Silva ${ }^{12}$, é o nome dado a toda a porção de um sistema com a qual um usuário mantém contato ao utilizá-lo, tanto ativa quanto passivamente. Assim, a interface do sistema precisa exibir de maneira clara e fácil para o profissional, onde estão os recursos que o mesmo precisa utilizar no dado momento. Uma interface que atenda a esse quesito é denominada de interface amigável ${ }^{13}$.

Diante do exposto, o objetivo deste artigo foi apresentar um sistema de software de gestão de clínica odontológica que além de cumprir com todos os requisitos quanto aos critérios de segurança, funcionalidade e interface, utiliza o recurso da filmagem como parte integrante do prontuário odontológico.

\section{Metodologia}

Inicialmente foram levantados os requisitos do sistema nomeado SIS Odonto. Esta tarefa foi realizada por discentes do curso de Sistemas de Informação, juntamente aos professores e alunos do curso de Odontologia, todos da Universidade Estadual do Sudoeste da Bahia (UESB). Os envolvidos tiveram como motivação produzir um sistema de software que atenda os critérios avaliados no estudo realizado por Oliveira ${ }^{6}$ (funcionalidade, segurança e interface), além de acrescentar o recurso da filmagem, servindo como prova legal em caso de um embate jurídico, envolvendo Cirurgião-Dentista e paciente.

Ao concluir a fase de levantamento de requisitos ficou definido que a primeira versão 
do programa contaria com o Gerenciamento de Pacientes, Funcionários e Usuários, Agenda do Consultório e do Dentista, Plano de Tratamento, Odontograma e Registro de Filmagem da Consulta.

Para o desenvolvimento deste sistema de software, foi escolhida a linguagem de programação Java em seu padrão SE (Standard Edition). Este padrão é usado para o desenvolvimento de aplicações destinadas a computadores pessoais, notebooks e arquiteturas com poder de processamento e memória consideráveis. A utilização do Java também possibilitou que a aplicação se tornasse multiplataforma, ou seja, pode ser instalada e utilizada em diversos sistemas operacionais ${ }^{14}$.

Não se optou por fazer um sistema Web, pois apesar de maior disponibilidade e facilidade de acesso proporcionada pela Internet, o volume de dados gerados pelos vídeos gravados durante cada consulta é muito alto. Dessa maneira tornou-se inviável construir o sistema online.

Os dados gerados são armazenados em um banco de dados relacional. Utilizou-se como Sistema Gerenciador de Banco de Dados (SGBD), o MySQL Server 5.5.28, mantido pela empresa Oracle Corporation. Este SGBD oferece rápidos recursos de multi-threaded e multiusuários, tornando-o bastante robusto ${ }^{15}$.

A seguir é apresentado o diagrama de caso de uso. Este descreve um cenário que mostra as funcionalidades do sistema do ponto de vista do usuário. A Figura 1 ilustra um diagrama de caso de uso. O "ator" mostrado no diagrama é um usuário com privilégios de atendente do consultório, tendo acesso apenas a funcionalidades básicas.

Figura 1. Diagrama de caso de uso mostrando quais recursos do sistema o usuário "Atendente" terá acesso.

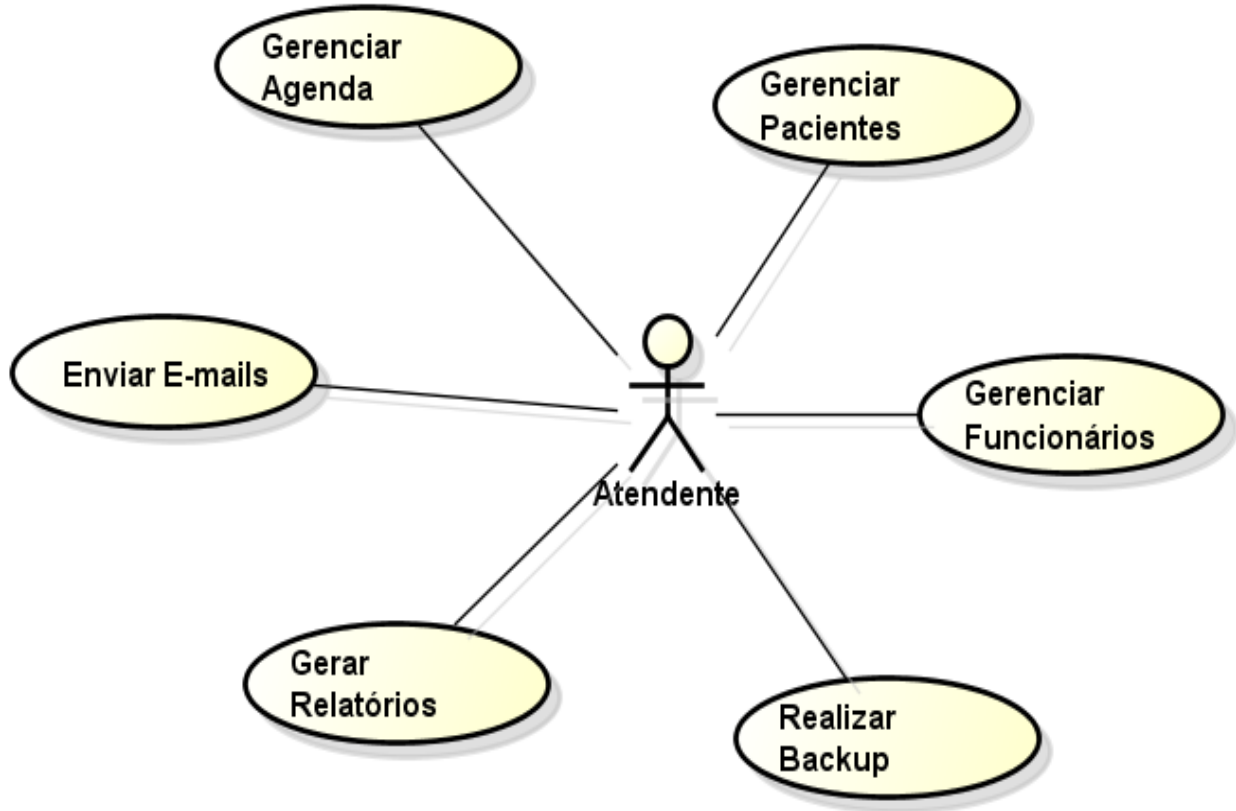

Com o intuito de aumentar a segurança e integridade do sistema, foi proposto controle de nível de acesso de acordo com o tipo de permissão. Desta maneira, cada usuário terá acesso apenas a informações relevantes a suas atribuições na clínica odontológica.

\section{Resultados e Discussões}

Foi desenvolvido um sistema de software, que atende aos quesitos de funcionalidade, segurança e interface, e ainda disponibiliza o recurso da filmagem para auxiliar o profissional no registro de tudo o que ocorre na interação profissional-paciente.

Para acessar o sistema de software o usuário deverá realizar o login com senha de acesso. Estes dados são cadastrados previamente pelo Administrador do sistema. As senhas de acesso dos usuários são criptografadas na base de dados ${ }^{16}$.

A Figura 2 ilustra o cadastro de paciente. Este formulário foi baseado no modelo de prontuário indicado pelo $\mathrm{CFO}^{7}$. Esta tela permite ao usuário cadastrar os dados pessoais do paciente, anexar uma foto e também inserir 
dados clínicos, através das abas "Anamnese" e "Exame Físico". Além disso, caso o paciente seja menor de idade, o programa provê o cadastro de seu responsável legal.

Figura 2. Tela de cadastro de pacientes

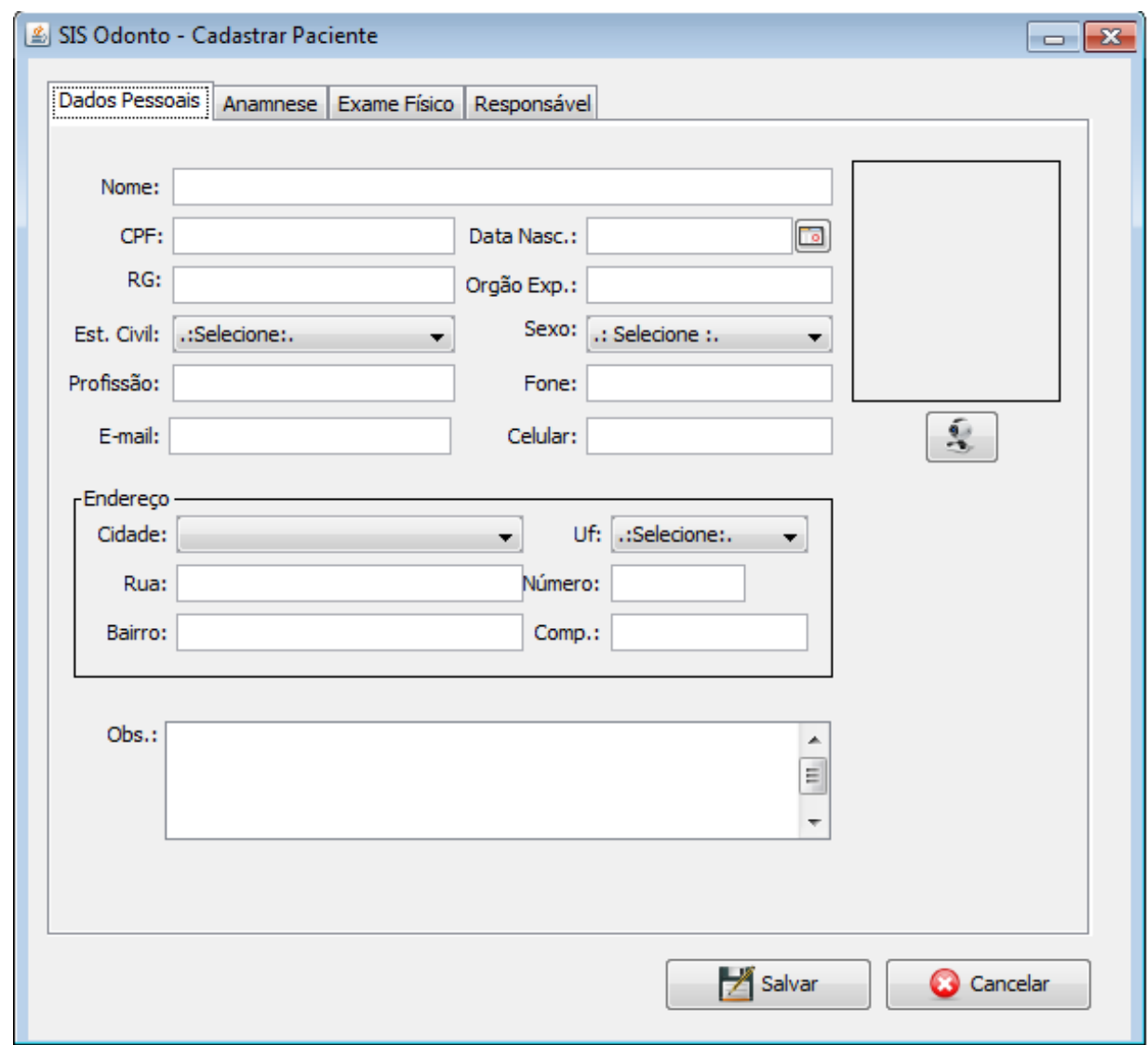

De acordo com Holanda et $\mathrm{al}^{17}$, a utilização de uma base de dados digital contendo informações agregadas, clínicas e administrativas em uma clínica odontológica são reconhecidas como grande impacto e benefício na melhoria da eficácia, eficiência, segurança e qualidade na prática da saúde.

O sistema provê a geração de diversos tipos de atestados, como se pode observar na Figura 3. Uma vez que o paciente esteja devidamente cadastrado na base de dados, o dentista poderá selecioná-lo e escolher qual tipo de atestado será gerado e o programa preencherá automaticamente os campos deste relatório. Essa foi uma significativa melhoria em relação à maioria dos sistemas de software existentes no mercado.

O estudo realizado por Oliveira ${ }^{6}$ mostrou que apenas $25 \%$ dos programas voltados para a gestão de clínicas odontológicas tem a possibilidade de gerar atestados.
Figura 3. Menu atestados

\begin{tabular}{|l|}
\hline Atestados \\
Atestado Escolar \\
Atestado Esportivo \\
Atestado de notificação compulsória \\
Atestado Militar \\
Atestado Trabalhista \\
\hline
\end{tabular}

Pode-se visualizar, através da Figura 4, a tela do odontograma. Nessa parte o CirurgiãoDentista poderá manipular de maneira simples todas as informações referentes à ficha clínica do paciente. A ficha clínica deve refletir não apenas os procedimentos clínicos realizados, mas também conter observações que se façam necessárias, como a falta de colaboração por parte do paciente, por exemplo. Dessa maneira, ao fim do tratamento serão analisados os resultados de acordo com todos os dados registrados ${ }^{18}$. 


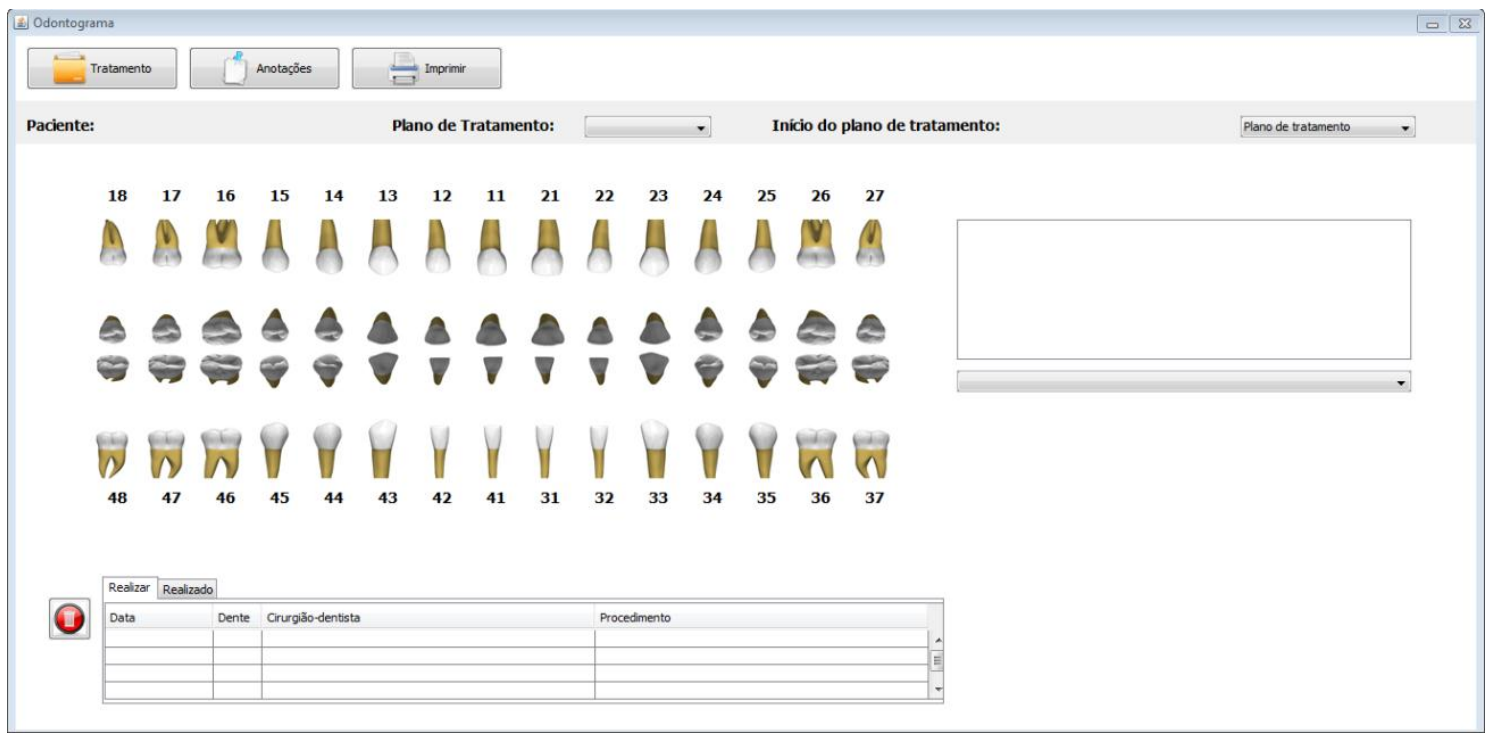

A ferramenta de backup (cópia de segurança) de dados, foi implementada como uma maneira de aumentar a segurança. O backup é de extrema importância para que se possa manter a integridade dos dados caso haja alguma falha no sistema, no hardware ou até mesmo para corrigir eventuais falhas de usuários, como por exemplo, a remoção acidental de um banco de dados. O sistema de backup foi programado no sistema JAVA para ser executado diariamente, no programa desenvolvido.

O sistema de software apresenta a funcionalidade de envio de e-mails para os pacientes e profissionais do consultório. Este instrumento pode ser utilizado para fortalecer o vínculo com os pacientes, por meio do envio de correspondências personalizadas, que podem ser para parabenizá-lo por aniversários ou datas especiais e também para lembrá-los de algum compromisso no consultório ${ }^{19}$. Além disso, podese utilizar este envio de mensagens eletrônicas como uma importante estratégia de marketing em um consultório odontológico.

O sistema de software também realiza a gestão de documentos dos pacientes. O Capítulo VII, artigo 17 do Código de Ética Odontológica obriga que se conserve todos os dados pertencentes ao prontuário odontológico em arquivo próprio seja na forma física ou digital. Além disso, segundo o Capítulo VII, artigo 18, parágrafo I, o odontólogo não pode negar ao paciente o acesso a seu prontuário, uma vez que seja solicitado pelo mesmo ${ }^{20}$.

Com relação ao tempo de guarda do prontuário odontológico, se apresenta como um assunto de extrema complexidade ${ }^{21}$. Entretanto,
Maciel et al. ${ }^{22}$ defende em seu estudo que a documentação odontológica deve ser guardada por tempo indeterminado permitindo que, em qualquer momento, todas as fases do plano de tratamento do paciente sejam consultadas, caso seja necessário.

A principal novidade apresentada pelo sistema é a possibilidade da filmagem das consultas e integração no prontuário odontológico, como pode ser visualizado na Figura 5. Antes de iniciar a consulta o paciente deverá autorizar por escrito a gravação. Yarid et al. ${ }^{8}$ afirmam que a filmagem tem valor como prova para demonstrar a conduta por parte do Cirurgião-Dentista nos procedimentos em que há necessidade de orientações ao paciente. Dessa forma, por ser capaz de registrar mais detalhes sobre toda a conversa formal e informal entre paciente e Cirurgião-Dentista, a filmagem como um documento legal, é capaz de armazenar inúmeras informações que deixam de ser registradas no prontuário, e, portanto, é capaz de preencher possíveis lacunas de informação na documentação odontológica convencional que podem ser exploradas em embates judiciais. Dessa forma, com a utilização da filmagem, profissional e paciente são amparados judicialmente.

Ao cadastrar o paciente, é criado um código de identificação, também chamado de Identification Code (ID), e uma pasta nomeada com este ID. O código de identificação é gravado no banco de dados e servirá para referenciar a pasta contendo as informações do paciente. Esta pasta é criada no disco rígido (HD - Hard Disk), interno ou externo ao computador. Dentro desta, 
são criadas 3 (três) subpastas com os nomes: Documentos, Imagens e Vídeos. O ultimo contém todas as gravações das consultas do pacientes. Então, o sistema de software necessita apenas do
ID do paciente, contido no banco de dados, para localizar a pasta, contendo o registro do paciente.

Figura 5. Tela de filmagem da consulta.

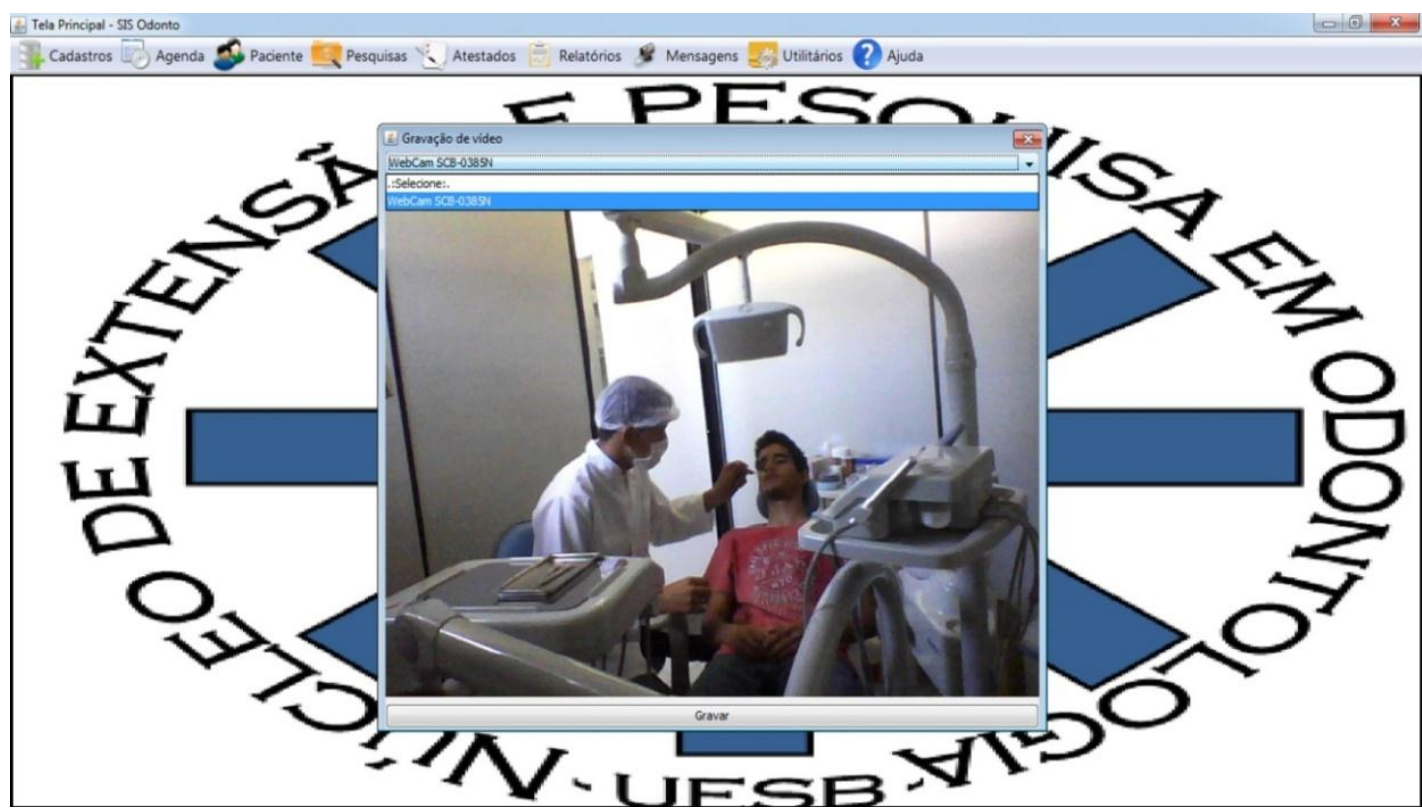

Quanto à comparação dos resultados obtidos com outros sistemas de software já comercializados, foram utilizados os mesmos métodos empregados por Oliveira ${ }^{6}$, no que se refere à funcionalidade, segurança e interface. As tabelas abaixo apresentam os sistemas de software avaliados pelo autor e a comparação destes com o SIS Odonto.
A Tabela 1 contém os valores percentuais atingidos por todos os sistemas de software analisados em relação aos itens obrigatórios. A Tabela 2 apresenta os valores percentuais alcançados por todos os sistemas de software avaliados em relação aos itens adicionais. A Tabela 3 mostra a segurança dos sistemas de software avaliados.

Tabela 1. Itens fundamentais presentes do SIS Odonto, em comparação com os sistemas avalizados por Oliveira ${ }^{6}$

\begin{tabular}{c|c}
\hline SOFTWARE & ITENS FUNDAMENTAIS \\
\hline SIS Odonto & $100 \%$ \\
\hline Easy Dental & $83,33 \%$ \\
\hline Dental Clinic & $50,00 \%$ \\
\hline Dental Office & $83,33 \%$ \\
\hline Dental Soft & $83,33 \%$ \\
\hline DocViewer Plus & $33,33 \%$ \\
\hline Odontologia Manager & $83,33 \%$ \\
\hline Open Dental & $83,33 \%$ \\
\hline Total Clinic Odontológico & $83,33 \%$ \\
\hline
\end{tabular}

Tabela 2. Itens suplementares presentes do SIS Odonto, em comparação com os sistemas avalizados por Oliveira ${ }^{6}$.

\begin{tabular}{c|c}
\hline SOFTWARE & ITENS SUPLEMENTARES \\
\hline SIS Odonto & $100 \%$ \\
\hline Easy Dental & $25,00 \%$ \\
\hline Dental Clinic & $25,00 \%$ \\
\hline Dental Office & $50,00 \%$ \\
\hline Dental Soft & $25,00 \%$ \\
\hline DocViewer Plus & $25,00 \%$ \\
\hline Odontologia Manager & $50,00 \%$ \\
\hline Open Dental & $25,00 \%$ \\
\hline Total Clinic Odontológico & $50,00 \%$ \\
\hline
\end{tabular}


Tabela 3. Itens de segurança dos softwares avaliados por Oliveira ${ }^{6}$ em comparação com o SIS Odonto.

\begin{tabular}{c|c|c|c}
\hline SOFTWARE & ACESSO POR SENHA & BACKUP & AUDITORIA \\
\hline SIS Odonto & $\mathrm{X}$ & $\mathrm{X}$ & $\mathrm{X}$ \\
\hline Easy Dental & $\mathrm{X}$ & $\mathrm{X}$ & $\mathrm{X}$ \\
\hline Dental Clinic & $\mathrm{X}$ & - & - \\
\hline Dental Office & $\mathrm{X}$ & $\mathrm{X}$ & $\mathrm{X}$ \\
\hline Dental Soft & $\mathrm{X}$ & $\mathrm{X}$ & - \\
\hline DocViewer Plus & - & - & - \\
\hline Odontologia Manager & - & $\mathrm{X}$ & - \\
\hline Open Dental & $\mathrm{X}$ & $\mathrm{X}$ & - \\
\hline Total Clinic Odontológico & $\mathrm{X}$ & $\mathrm{X}$ & - \\
\hline
\end{tabular}

A avaliação da interface foi baseada nas heurísticas propostas por Nielsen ${ }^{23}$. As heurísticas, de acordo com Nielsen, são regras gerais para descrever as propriedades das interfaces utilizáveis.

Existem diversas recomendações propostas para garantir a usabilidade de um sistema. Nielsen ${ }^{23}$ propôs 10 heurísticas de usabilidade que um sistema deve garantir. Ainda que existam outras recomendações de outros autores, as Heurísticas de Nielsen são largamente utilizadas por organizações em todo o mundo, principalmente para construção de interfaces de sistema de software e websites.
$\mathrm{Na}$ lista de heurísticas de usabilidade proposta por Nielsen, a interface de um sistema de computador deve conter: visibilidade do estado do sistema, relacionamento entre a interface do sistema e o mundo real, liberdade e controle do usuário, consistência e padronização, prevenção de erros, reconhecimento ao invés de lembrança, flexibilidade e eficiência de uso, estética e design minimalista, auxílio aos usuários, diagnosticar e sanar erros, e ajuda e documentação. A Tabela 4 ilustra a avaliação das interfaces dos sistemas de software analisados.

Tabela 4. Avaliação da interface dos softwares avaliados por Oliveira ${ }^{6}$ em comparação com o SIS Odonto.

\begin{tabular}{c|c}
\hline SOFTWARE & HEURÍsTICAS \\
\hline SIS Odonto & $100 \%$ \\
\hline Easy Dental & $60,00 \%$ \\
\hline Dental Clinic & $50,00 \%$ \\
\hline Dental Office & $80,00 \%$ \\
\hline Dental Soft & $50,00 \%$ \\
\hline DocViewer Plus & $50,00 \%$ \\
\hline Odontologia Manager & $20,00 \%$ \\
\hline Open Dental & $20,00 \%$ \\
\hline Total Clinic Odontológico & $80,00 \%$ \\
\hline
\end{tabular}

\section{Conclusão}

O sistema de software desenvolvido cumpriu os requisitos de funcionalidade, segurança e interface, e apresentou melhorias em relação aos demais sistemas existentes no mercado considerando os requisitos avaliados.

Este sistema também apresentou algumas ferramentas que o distingue dos demais. É importante destacar o recurso da filmagem como parte integrante do prontuário odontológico, servindo como prova legal em caso de um embate jurídico envolvendo Cirurgião-Dentista e paciente.

Como trabalhos futuros, pretende-se desenvolver uma segunda versão do sistema, incluindo módulos de gerenciamento de estoque e de finanças. Mais testes serão realizados para corrigir possíveis falhas de execução do sistema de software, e por fim pretende-se implantar o sistema de software na clinica de odontologia da Universidade Estadual do Sudoeste da Bahia (UESB), e obter a avaliação do sistema desenvolvido por profissionais da Odontologia para analisar os benefícios alcançados em um ambiente real. 


\section{Referências}

1. Roman DJ, Osinski M, Erdmann R. H. A substantive theory on the implementation process of operational performance improvement methods. Revista de Administração (FEA-USP). 2016; doi: 10.1016/j.rausp.2016.12.005.

2. Albuquerque JP, Nerling $M$, Prado EPV. Gestão da Tecnologia da Informação em hospitais: uma avaliação das unidades de saúde do município de Guarulhos. 8o CONTECSI International Conference on Information Systems and Technology Management; 2011; São Paulo, Brasil. Proceedings of the 8th CONTECSI International Conference on Information Systems and Technology Management; 2011. p. 31093121.

3. Bates DW, Gawande AA. Improving safety with Information Technology. The New England Journal of Medicine. 2003; 348(25):2526-2534.

4. Jordi L, Figueiredo MÇ, Barone D, Pereira C. Study and analysis of information technology in dentistry in Latin American countries. Acta Odontológica Latinoamericana. 2016; 29(1): 1422.

5. Kubo CS, Júnior MSA. Os sistemas aplicativos (softwares) no consultório odontológico. Revista Internacional de Aprendizaje y Cibersociedad. 2016; 18(2): 65-70.

6. Oliveira US, Costa ACS, Yarid SD, Santos $A F$, Filho IEM, Silva CA. et al. Technical analysis of softwares used for dental practice management. Revista Sul-Brasileira de Odontologia. 2012 OctDec;9(4):394-400.

7. Almeida CAP, Zimmermann RD, Cerveira JGV, Julivaldo FSN. Prontuário odontológico uma orientação para o cumprimento da exigência contida no inciso VIII do art. 5.ㅇ do Código de Ética Odontológica. Rio de Janeiro: CFO; 2004.

8. Yarid SD, Peres SHCS, Peres AS, Lauris JRP, Bastos JRM. Documentação odontológica aceita como prova legal por Juízes de Varas Cíveis Brasileiras. Ver Assoc Paul Cir Dent 2008; 62(6):488-93.

9. Morris R, Thompson K. Password security: a case history. Communications of the ACM. 1979; 22(11): 594-597.

10. Silva DRP, Stein LM. Segurança da informação: uma reflexão sobre o componente humano. Ciências \& Cognição 2007; Vol 10: 4653.

11. Chatterjee S, Sarker S, Valacich JS. The behavioral roots of information systems security:
Exploring key factors related to unethical IT use. Journal of Management Information Systems. 2015. 31(4): 49-87.

13. Barbosa SDJ, Silva BS. Interação humano-computador. Rio de Janeiro: Elsevier; 2010.

14. Ferreira SBL, Leite JCSP. Avaliação da Usabilidade em Sistemas de Informação: o Caso do Sistema Submarino. RAC. 2003. Abr./Jun; 7(2): 115-136.

15. Deitel PJ, Deitel HM. Java, How to Program. 10a edition. Pearson: Upper Saddle River, New Jersey; 2015.

16. Oracle Corporation. MySQL 5.5 Reference Manual [internet]. c1997-2013 [acesso em 2017 Março 11]. Disponível em: https://dev.mysql.com/doc/relnotes/mysql/5.5/ en/

17. Smart NP. Cryptography Made Simple (Information Security and Cryptography). 1a edition. Springer. 2015.

18. Holanda DA, Mello VVC, Zimmermann RD. Documentação Digital em Odontologia. Revista Odontologia Clinica Científica. 2010; 9(2):111-113.

19. Silva M. Documentação em Odontologia e sua importância jurídica. Revista Odontologia e Sociedade. 1999; 1(1):1-3.

20. Zuchini ARB, Lolli MCGS, Lolli LF, Lolli HA. Perfil profissional do cirurgião-dentista em associação ao conhecimento e utilização de marketing. Arquivos em Odontologia. 2016; 48(1): 19-25.

21. Conselho Federal de Odontologia. Código de Ética Odontológica. Rio de Janeiro: CFO; 2003.

22. Saraiva AS. A importância do prontuário odontológico - com ênfase em documentos digitais. Revista Brasileira de Odontologia. 2011; 68(2):157-160.

23. Maciel SML, Xavier YMA, Leite PHAS, Alves PM. A documentação odontológica e a sua importância nas relações de consumo: um estudo em Campina Grande-PB. Pesquisa Brasileira Em Odontopediatra e Clinica Integrada. 2003; $3(2): 53-8$.

24. Nielsen J. Usability engineering. Cambridge: Academic Press; 1993. 
Endereço para Correspondência

Universidade Federal do Recôncavo da Bahia UFRB

Av. Rui Barbosa, no 710, Cruz das Almas - BA

CEP.: $44380-000$

e-mail: alexferreira.ba@gmail.com

Recebido em 24/04/2017

Aprovado em 09/11/2017

Publicado em 30/03/2018 